\title{
New Correlation Ratio in Chemistry and Biology with Respect to Time and Magnetic Field
}

\author{
Aibassov Yerkin Zhakenovich*, Yemelyanova Valentina, Tussupbayev Nessipbay, Shakieva Tatyana, Abenov \\ Bakhyt and Yerzhanova Zhadyra \\ Research Institute of New Chemical Technologies and Materials, Kazakh National University Al-Farabi, Almaty 005012, Kazakhstan
}

Abstract: The authors reviewed the influence of various factors - inductive, mesomeric and steric effects, and magnetic fields and time correlation ratio. The authors offered the new correlation ratio in Chemistry and Biology with respect to time and magnetic field. The proposed formula takes into account the influence of the magnetic field on the correlation ratio.

Key words: Magnetic field, time, correlation ratio.

\section{Introduction}

At present, the rapid development of science, technology and equipment allows us to look at the classical correlation ratio of FEL (free energy linearity) with new prospects [1-7].

An urgent problem is the question of how to conduct the correlation ratio linearity of free energy in the presence of a magnetic field. Addressing these issues is a scientific and practical significance.

Objective considers how to behave correlation ratio linearity of free energies under the influence of a magnetic field.

\section{Theory}

Correlation ratios characterize empirically established relationship between different properties of some chemical compounds (reactivity, physical, thermodynamic properties, biological activity, and others) and structure parameters, medium or properties of the same or a different number of compounds. Formal prototype of most of the correlation ratios-equation Brënsteda expressing experimentally found a linear relationship between the reaction rate constant $k_{c}$ acid (base) catalysis and dissociation equilibrium constants acid (base) $K_{a}$ :

\footnotetext{
${ }^{*}$ Corresponding author: Aibassov Yerkin Zhakenovich, professor, research field: organic chemistry of $\mathrm{U}, \mathrm{Th}, \mathrm{As}, \mathrm{Sb}$, Bi. E-mail: erkin53@mail.ru.
}

$$
\lg k_{e}=a \lg K_{a}+c
$$

where, $a$ and $c$-Empirical parameters for the reaction. Eq. (1)-example of the overall ratio of FEL, according to the change in free reaction energy $\left(\mathrm{DG}^{\circ}\right)$ and the free energy of activation $\left(\mathrm{DG}^{++}\right)$, which caused various reactions in the same structure variations reactants or the medium is linear:

$$
d_{R} D G_{i}=a d_{R} D G_{j}+b
$$

where, $d_{R}$-an operator changing the structure (e.g., introduction of a substituent at $R$ ) or medium, $i, j-$ reactions indices.

Hammett equation that associates changes in velocity or equilibrium constants induced by introduction of substituent into the aromatic benzene ring in the compound I, with changes in the dissociation constants of the benzoic acid II with a similar substitution:

$$
d_{R} D G_{i}=a d_{R} D G_{B A}^{0}
$$

where, $D G_{i}$ - the free energy of activation of reaction or any reaction of a compound I in arbitrary (but identical to this series of compounds) conditions, and $\mathrm{DG}_{\mathrm{BA}}^{0}$ - free energy of dissociation of benzoic acid in the set (not necessarily identical) conditions. Eq. (3) reduces to:

$$
\lg \left(k / k_{0}\right)=r \lg \left(K / K_{0}\right)_{B A}
$$

where, $k$-rate constant (equilibrium) reaction of compound I with a substituent $R$, and $k_{0}$ is the same constant for compounds with $R=\mathrm{H}, K$ and 
$K_{0}$-dissociation constants corresponding benzoic acid II, $r$-constant characterizing the relative (in comparison with the reference series dissociation benzoic acid) compound I sensitivity reactions to structural changes.

Series dissociation reaction of benzoic acids in water at $25{ }^{\circ} \mathrm{C}$ is chosen as the standard, and are constants of the substituents $R$ the value of $s=$ $\lg \left(K / K_{0}\right)$. Eq. (4) is known as the Hammett Equation and is typically written in the following form:

$$
\lg k=r s+\lg k_{0}
$$

Eq. (5) allows the calculation of kinetic and equilibrium parameters of many reactions of benzene derivatives with meta and para substituent, having constants of substituents and variables $r$, defined statistically significant for several compounds of the reaction series. Correlation ratios for structural variations of the substrate was researched. The Table 1 shows the values of the constants $\mathrm{s}$ deputies for some of the most important groups $\left(\sigma_{\mathrm{m}}\right.$ and $\sigma_{\mathrm{p}}$ - constants of the substituents in the meta and para positions). For reactions accelerated by electron-donating substituents $(\mathrm{s}<0), \mathrm{r}<0$, for reactions accelerated by electron-withdrawing substituents.

Correlation four-parameter Eq. (6):

$$
\lg \left(k / k_{0}\right)=\rho_{I} \sigma_{I}+\rho_{I}^{o} \sigma_{C}^{o}
$$

allowing of separately evaluate the contributions of induction and mesomeric effects of substituents on the relative reactivity. There are other correlation ratio [Yokawa-Tsuno, Dewar-Grisdeyla, Sven (Svein)-Lupton], in which a different approach to the division of the general effect of the substituent on its components. Development of the correlation ratio for the characterization of the reactivity of the other types of connections-polynuclear aromatic, heterocyclic, olefins and aliphatic compounds. In the latter case, the equation used Taft $\left[s^{*}\right.$-constant Taft, $k$ and $k_{\mathrm{CH} 3}$ - velocity or equilibrium constants of reactions of aliphatic and $\mathrm{RY}$ respectively $\mathrm{CH}_{3} \mathrm{Y}, r^{*}$-parameter analogous $r$ in Eq. (4)]:

$$
\lg \left(k / k_{\mathrm{CH} 3}\right)=\rho^{*} \sigma^{*}
$$

and for compounds with multiple substituents:

$$
\lg \left(k / k_{\mathrm{CH} 3}\right)=\rho^{*} \Sigma \sigma^{*}
$$

Table 1 The constants of the substituents $R$ for different groups.

\begin{tabular}{llllllllll}
\hline $\mathrm{R}$ & $\sigma_{\mathrm{m}}$ & $\sigma_{\mathrm{p}}$ & $\sigma^{+}$ & $\sigma^{-}$ & $\sigma_{\mathrm{I}}$ & $\sigma_{\mathrm{C}}$ & $\sigma_{\mathrm{CH} 2 \mathrm{R}}^{*}$ & $\mathrm{E}$ & $\chi_{\mathrm{M}, 10^{4}}$ \\
\hline $\mathrm{H}$ & 0 & 0 & 0 & 0 & 0 & 0 & 0 & 0 & -2.93 \\
$\mathrm{~N}\left(\mathrm{CH}_{3}\right)_{2}$ & -0.21 & -0.83 & -1.5 & - & 0.06 & -0.94 & - & - & - \\
$\mathrm{NH}_{2}$ & -0.16 & 0.66 & -1.3 & - & 0.12 & -0.78 & - & - & - \\
$\mathrm{OCH}_{3}$ & 0.12 & -0.27 & -0.78 & - & 0.27 & -0.63 & 0.52 & - & -3.36 \\
$\mathrm{CH}_{3}$ & -0.07 & -0.17 & -0.31 & - & -0.04 & -0.07 & -0.10 & -0.07 & -6.0 \\
$\mathrm{C}_{2} \mathrm{H}_{5}$ & -0.07 & -0.15 & -0.30 & - & - & -0.07 & -0.12 & -0.36 & - \\
$\mathrm{i}-\mathrm{C}_{3} \mathrm{H}_{7}$ & -0.07 & -0.15 & -0.28 & - & - & -0.08 & - & -0.09 & - \\
$\mathrm{t}-\mathrm{C}_{4} \mathrm{H}_{9}$ & -0.10 & -0.20 & -0.26 & - & - & -0.09 & -0.17 & -1.74 & - \\
$\mathrm{C}_{6} \mathrm{H}_{5}$ & 0.06 & -0.01 & -0.18 & - & 0.10 & -0.10 & 0.22 & -0.38 & - \\
$\mathrm{F}$ & 0.34 & 0.06 & -0.07 & - & 0.50 & -0.59 & 1.10 & -0.24 & -6.3 \\
$\mathrm{Cl}$ & 0.37 & 0.23 & 0.11 & - & 0.46 & -0.35 & 1.05 & -0.24 & -20.1 \\
$\mathrm{Br}$ & 0.39 & 0.23 & 0.15 & - & 0.44 & -0.34 & 1.00 & -0.27 & -30.6 \\
$\mathrm{~J}$ & 0.35 & 0.28 & 0.14 & - & 0.39 & -0.23 & 0.85 & -0.37 & -44.6 \\
$\mathrm{COOCH}_{3}$ & 0.37 & 0.45 & - & 0.64 & 0.30 & 0.03 & - & - & 0.8 \\
$\mathrm{COCH}_{3}$ & 0.38 & 0.50 & - & 0.84 & 0.28 & 0.05 & 0.60 & - & 1.73 \\
$\mathrm{CN}$ & 0.56 & 0.66 & - & 0.88 & 0.56 & 0 & 1.30 & - & -2.8 \\
$\mathrm{NO}_{2}$ & 0.71 & 0.77 & - & 1.24 & 0.65 & 0 & - & - & -2.11 \\
$\mathrm{~N}^{+}\left(\mathrm{CH}_{3}\right)_{3}$ & 0.88 & 0.82 & 0.41 & 0.77 & 0.91 & 0 & 1.90 & - & - \\
$\mathrm{CF}_{3}$ & 0.43 & 0.54 & - & 0.65 & 0.45 & 0 & - & - & -24.9 \\
$\mathrm{SO}_{2} \mathrm{CH}_{3}$ & 0.60 & 0.72 & - & 0.98 & 0.59 & 0 & - & -11.23 \\
\hline
\end{tabular}


and $s^{*}$ is linearly related to the constants $s_{I}$ :

$$
s^{*}(R)=6.23 s_{I}(R), s_{I}(R)=0.45 s^{*}\left(\mathrm{CH}_{2} \mathrm{R}\right)
$$

To account for the influence of the spatial effects of substituents on the reactivity of aliphatic compounds are introduced steric constants of substituents Es, which defined according to the speed of the acid hydrolysis of esters:

$$
E_{s}=\lg \left(k / k_{0}\right)
$$

where, $k$ and $k_{0}$-rate constants, respectively, for compounds with $R$ substituent in acyl- and acetatecomponents. Es constants determined by the amount of substituents and are linearly dependent on their van der Waals radii. Joint account of the influence of electronic and steric factors on the reactivity of aliphatic compounds is carried out by means of the Eq. (9):

$$
\lg \left(k / k_{0}\right)=r^{*} s^{*}+r_{s} E_{s}
$$

where, $r_{s}$-parameter analogous to the sense of $r$ in Eq. (4).

For bimolecular nucleophilic substitution is performed Sven-Scott equation:

$$
\lg \left(k / k_{0}\right)=\sin
$$

where, $k$ and $k_{0}$ - the reaction rate constant of the substrate, respectively, with the water and the nucleophile $s$ parameter characterizes the response sensitivity to variations in reactant (selectivity), the constant $n$ is determined from the data on reaction kinetics of a standard substrate-a number of nucleophiles $\mathrm{CH}_{3} \mathrm{Br}$ and depend the reaction conditions (medium, temperature). For aqueous solutions at $0{ }^{\circ} \mathrm{C}$, for some values of $n$ equal nucleophiles 6.35 (for $\left.\mathrm{S}_{2} \mathrm{O}_{3}{ }^{2-}\right), 5.13\left(\mathrm{CN}^{-}\right), 4.16\left(\mathrm{NH}_{3}\right)$, $2.99\left(\mathrm{Cl}^{-}\right), 1.88\left(\mathrm{~F}^{-}\right)$, and $0\left(\mathrm{H}_{2} \mathrm{O}\right)$. For reactions of stable organic cations and activated carbonyl compounds with nucleophiles good correlation using the equation is achieved by Ritchie:

$$
\lg k=N_{+}+C
$$

where, $C-$ a constant characteristic of the substrate.

For the velocity correlation equation used solvolysis Grunwald-Uinsteyn:

$$
\lg \left(k / k_{0}\right)=m Y
$$

where, $m$-constant sensitivity of similar reactions. constant $r$ in Eqs. (4-9), and $Y$-parameter of solvent polarity. As a standard series $(\mathrm{m}=\mathrm{I})$ is selected solvolysis tert $-\mathrm{C}_{4} \mathrm{H}_{9} \mathrm{Cl}$ in $80 \%$ ethanol. For a wider range of reactions used multiparameter correlation ratio, including solvent parameters calculated on the basis of spectral data (displacement of long-wavelength absorption bands of polar Port in various solvents) — Dimroth FT, Kosovera constant Z, constant Taft-Kamle $\mathrm{p}^{*}$. The basis of the correlation ratios for the physical characteristics of quantum-mechanical calculations is installed linear dependence of the constants of substituents and the quantities of electronic charge induced substituent on the reaction center and adjacent atoms, and other characteristics of the electron distribution in molecules and ions. There are a variety of correlation relationships between the dipole moments, the frequency and intensity of the bands in the vibrational spectra, chemical shifts in the NMR (nuclear magnetic resonance) spectra, etc. of various types of electronic and steric constants of substituents. For the chemical shifts in the 19F NMR spectra of metha- $\left(F_{m}\right)$ and para-substituted $\left(\mathrm{F}_{\mathrm{p}}\right)$ benzenes with a good degree of accuracy the correlation ratio:

$$
\begin{aligned}
& \delta\left(F_{m}\right)=5.26 \sigma_{I}+0.81 \sigma_{C}^{o} \\
& \delta\left(F_{p}\right)=7.02 \sigma_{I}+30.55 \sigma_{C}^{o}
\end{aligned}
$$

For the intensity fluctuations of benzene rings in the 1,600 and $1,585 \mathrm{~cm}^{-1}$, characterizing distortion p-electron system of the ring is performed correlation ratio:

$$
(A-100)^{1 / 2}=132.7 s_{c}
$$

where, $A$ - the integrated intensity of the absorption band in the IR (infrared) spectrum.

The correlation ratio in biochemistry is an important means of targeted search prediction structural modifications that improve biological activity in a series of compounds with a varying systematic structural feature.

An important parameter to associate with the biological activity - distribution coefficient PB binary 
hydrocarbon-water system. $P$ values determined for more than 10,000 compounds. The total value for the correlation model of "structure-activity" is based on the study of relations (15)-(17):

$$
\text { Biological response speed }=B C k
$$

where, $C$-amount of drug injected into the body, in the factor characterizing the probability of achieving a particular drug receptor for a period of time, $k$ is a constant velocity or equilibrium limiting reaction steps leading to the controlled biological response. Eq. (15) is reduced to the correlation ratio:

$$
\lg (1 / C)=a(\lg P)^{2}+b(\lg P)+c \lg k+d(16)
$$

where, $a, b, c, d$-factor obtained in the result of statistical processing of the experimental data. For many biological systems for the constant $k$ is performed correlation ratio:

$$
l g k=a p+b v+c E_{s}+d
$$

where, $p=\lg (P / \mathrm{pH}), \mathrm{pH}-\mathrm{a}$ distribution ratio for the connection with the deputy. Eqs. (15-17) -an important element of the system analysis of the biological activity of organic and natural product.

\section{Results and Discussion}

Inductive effect (polar effect) - the displacement of the electron density of the chemical bond of $\sigma$-bonds. It is a kind of field effect. The concept of inductive effect was introduced by Ingold, they were introduced notation:

- $+\mathrm{I}-$ effect in the case of increasing the electron density deputy;

- -I-effect in the case of lowering the electron density substituent.

As a matter of comparison take unsubstituted compound, that is, zero inductive effect is taken for the hydrogen atom. A characteristic feature of the inductive effect compared to mesomeric effect is its rapid decay of the chain links. Among the most distinctive + I-groups are: alkyl group metals metalloid group (silyl, boron, phosphorus and so on); among the most typical groups -I-effect allocated charged groups (because the field-effect), such as trialkyl ammonium, dialkylsulfone and other onium salts, nitro, hydroxy, alkoxy, amino, halogen and m. p.

The effect is due primarily atom to which the source is directly linked carbon atom is thus determined by the difference in electronegativity atoms. Quantification of inductive effect can be made using the equation Taft.

Mesomeric effect (coupling effect, resonance effect) - the displacement of the electron density of the chemical bond of $\pi$-bonds. It explains the theory of chemical resonance.

The emergence of mesomeric effect related to the change profile $\pi$-system molecule. Background $\pi$-system overlaps with $\pi$-system of p-orbitals or substituent, whereby there is a redistribution of electron density. It is the latter detects the presence of $+\mathrm{M}$ or $-\mathrm{M}$ effect. The sign, as in the case of an inductive effect is determined by adding the electron density and is opposite the Deputy parameter $\sigma$ in the equation Hammett.

In contrast to the inductive effect mesomeric effect weakly damped system of connections, with the only difference that in this case requires a system of multiple bonds and p-orbitals.

The most characteristic $+\mathrm{M}$ groups are those which introduce unshared electron pairs in a conjugate system, such as amino, hydroxy, alkoxy, halogen.

Groups with -M-effect contains multiple bonds to hetero. An analogy can be different types of nitrogen atoms in the heterocycles: 1) For the $+\mathrm{M}$ characteristic hetero pyrrole; 2) For -M characterized pyridine heteroatoms. -M group is a carbonyl group, a cyano group and m.p.

Groups with a system of conjugated carbon-carbon bonds, such as phenyl and vinyl groups occupy an intermediate position of the so-called $\pm \mathrm{M}$-effect and manifest effect, the opposite effect of the group, which are connected to amplify.

Steric effect - the effect of the spatial volume of the molecules in the course of a chemical reaction. Thus, the presence of large groups in the molecule close 
to the reacting atoms may prevent convergence of these atoms and slow reaction or make it impossible.

Thus, taking into account the motion of an electron in a magnetic field, Schrödinger and Dirac in the magnetic field, the authors concluded that the inductive, mesomeric and steric effects can be combined overall correlation ratio.

The authors offer new correlation ratio in chemistry and biology with the time and the magnetic field:

$$
\log K= \pm I+ \pm M+ \pm S+B+\Delta \tau
$$

where, $I$-inductive effect, $M$-mesomeric effect, $S$-steric effects, $B$-magnetic effect, $\Delta \tau$-time.

\section{Conclusions}

Using the methods of correlation analysis carried out a systematic study of the influence of the nature of the various components of the equilibrium constant. The influence of the nature of the reagents in the reaction equilibrium of various types have been studied. It is proved that the impact of laws on the nature of the reagents and all they subject to constant distribution principle FEL and allow to quantify the influence of the diluents in all types of systems.

The authors proposed a new correlation coefficient in chemistry and biology, which takes into account the inductive, mesomeric, steric effects, and the effects of time and magnetic field.

It established a large number of one-parameter linear correlations describing the influence of the nature of the reagents in the reaction equilibrium. Found multivariate linear correlations, describing the joint effect of the structure and nature of the reagents extractants containing cross-members in the case of the mutual influence of the studied factors.

\section{References}

[1] Zhdanov, Y., and Minkin, V. I. 1966. Correlation Analysis in Organic Chemistry. Rostov: Rostov University Publishing.

[2] Palm, V. A. 1967. Basics Quantity Theory of Organic Reactions. Sant Petersburg: Petersburg University.

[3] Johnson, K. 1977. Hammett Equations. Moskva: Moscow University Press.

[4] Exner, O. 1972. Advances Inlinear Free Energy Relationships. ed. by Chapman, N. B., and Shorter, J. N.Y.: Wiley.

[5] Ewing, D. F. 1978. Correlation Analysis in Chemistry. Recent Advances. ed. by Chapman, N. B., and Shorter, J. N.Y.: Wiley.

[6] Hansch, C., and Leo, A. 1979. Substituent Constants for Correlation Analysis in Chemistry and Biology. N.Y.: Wiley.

[7] Klumpp, G .W. 1982. Reactivity in Organic Chemistry. N.Y.: Wiley. 